\title{
Papers
}

\section{Meta-analysis of effects and side effects of low dosage tricyclic antidepressants in depression: systematic review}

Toshi A Furukawa, Hugh McGuire, Corrado Barbui

\begin{abstract}
Objective To compare the effects and side effects of low dosage tricyclic antidepressants with placebo and with standard dosage tricyclics in acute phase treatment of depression.

Design Systematic review of randomised trials comparing low dosage tricyclics ( $\leqslant 100 \mathrm{mg}$ /day) with placebo or with standard dosage tricyclics in adults with depression.

Main outcome measures Relative risk of response in depression (random effects model), according to the original authors' definition but usually defined as $50 \%$ or greater reduction in severity of depression. Relative risks of overall dropouts and dropouts due to side effects.

Results 35 studies (2013 participants) compared low dosage tricyclics with placebo, and six studies (551 participants) compared low dosage tricyclics with standard dosage tricyclics. Low dosage tricyclics, mostly between 75 and $100 \mathrm{mg}$ /day, were 1.65 (95\% confidence interval 1.36 to 2.0 ) and 1.47 (1.12 to 1.94 ) times more likely than placebo to bring about response at 4 weeks and 6-8 weeks, respectively. Standard dosage tricyclics failed, however, to bring about more response but produced more dropouts due to side effects than low dosage tricyclics.

Conclusions Treatment of depression in adults with low dose tricyclics is justified. However, more rigorous studies are needed to definitively establish the relative benefits and harms of various dosages.
\end{abstract}

\section{Introduction}

Despite the growing popularity of selective serotonin reuptake inhibitors and other newer antidepressants, tricyclic andtidepressants are still extensively prescribed worldwide. In the United Kingdom between 1991 and 1996, there was a $460 \%$ increase in prescriptions for selective serotonin reuptake inhibitors, but there was also a $40 \%$ increase in prescriptions for tricyclics for patients starting treatment, with these new patients still outnumbering those taking selective serotonin reuptake inhibitors by $56 \% .{ }^{1}$ In the United States between 1990 and 1995 antidepressant use increased by $73 \%$ mainly because of patients being prescribed selective serotonin reuptake inhibitors, but even today tricyclics are prescribed as often as selective serotonin reuptake inhibitors. ${ }^{23}$ Other countries show similar trends. ${ }^{4}$
Evidence for the recommended dosage of tricyclics is poor. ${ }^{5}$ Many of the existing guidelines recommend dosages greater than $100 \mathrm{mg}$ /day or $125 \mathrm{mg}$ /day, but there is a lack of convincing evidence that lower dosages are not effective. ${ }^{78}$ This uncertainty casts doubt on the widely held view that depression is undertreated both in primary care and in psychiatric settings. ${ }^{9}{ }^{10}$ It also questions whether selective serotonin reuptake inhibitors should be preferred over tricyclics when controlled trials failed to find differences in effectiveness between the two, because it is easier to achieve "adequate" dosage with selective serotonin reuptake inhibitors. ${ }^{11}$

\section{Methods \\ Inclusion criteria}

We included randomised trials comparing low dosage tricyclics with placebo or with standard dosages of the same tricyclic in the acute phase treatment of adults with depression. Low dosage was defined as $100 \mathrm{mg}$ /day or less of imipramine, amitriptyline, clomipramine, desipramine, doxepin, dothiepin, trimipramine, or lofepramine. We excluded nortriptyline because the standard dosage is debatable. Standard dosage was defined as greater than $100 \mathrm{mg}$ /day. Our trial was to last at least four weeks.

Our primary outcome was the effect of treatment on depression, according to the original authors' definition but usually defined as $50 \%$ or greater reduction in severity of depression. The severity of symptoms was measured by either observer rating (preferred) or self report.

\section{Identification of trials}

We electronically searched the Cochrane Collaboration depression, anxiety, and neurosis controlled trials register up to November 2000 for any trials in which tricyclics were given. This database incorporates results of group searches of Medline (1966 onwards), Embase (1980 onwards), CINAHL (1982 onwards), PsycINFO (1974 onwards), PSYNDEX (1977 onwards), and LILACS (1982-99). We also hand searched the major psychiatric and medical journals. Two reviewers (HM and TAF) then manually examined the potential papers to see if they were randomised trials comparing low dosage tricyclics with placebo or with standard dosage for any form of depression. All potential identified papers were then

\author{
Department of \\ Psychiatry, Nagoya \\ City University \\ Medical School, \\ Nagoya 467-8601, \\ Japan \\ Toshi A Furukawa \\ professor \\ Cochrane \\ Collaboration \\ Depression, \\ Anxiety, and \\ Neurosis, Health \\ Services Research, \\ King's College \\ Institute of \\ Psychiatry, London \\ SE5 8AF \\ Hugh McGuire \\ trials search \\ coordinator \\ Department of \\ Medicine and \\ Public Health, \\ Section of \\ Psychiatry, \\ University of \\ Verona, Ospedale \\ Policlinico 37134 \\ Verona, Italy \\ Corrado Barbui \\ psychiatrist \\ Correspondence to: \\ T A Furukawa \\ furukawa@med. \\ nagoya-cu.ac.jp
}

bmj.com 2002;325:991 
checked according to the strict eligibility criteria by two independent reviewers (TAF and $\mathrm{CB}$ ).

To identify further reports TAF checked the references of this preliminary list of selected studies along with references of other relevant review papers. To identify more recent reports HM subjected nine of the most representative studies to SciSearch. TAF contacted authors of major papers and other experts in the specialty.

\section{Quality assessment and data extraction}

TAF and CB assessed the methodological quality of the selected studies. The criteria for quality assessment were based on the recommendations of the Cochrane Collaboration Handbook and focused on concealment of allocation and double blinding. ${ }^{12} \mathrm{HM}$ and TAF independently extracted data from the original reports using data extraction forms. Disagreements between the two reviewers were resolved by consensus.

\section{Statistical analysis}

Data were entered twice into Review Manager (version 4.1) using the duplicate data entry facility. For dichotomous outcomes, we calculated relative risks and their $95 \%$ confidence intervals with a random effects model because they may be more generalisable and more easily interpreted than those obtained with fixed effects models, odds ratios, or risk differences. ${ }^{13}{ }^{14}$ We assessed heterogeneity between studies with the $Q$ statistic and by visual inspection of the results. For continuous outcomes, we calculated standardised weighted mean differences with a random effects model.

We first performed per protocol analysis according to the values reported by the original authors. When data on dropouts were included by the last observation carried forward method, we analysed them according to the primary studies. We also performed a worst case scenario intention to treat analysis whereby dropouts were considered non-responders in the active treatment group but as responders in the placebo group. This extreme scenario was to guard against favouring active drugs that could be the more harmful.

We performed a funnel plot analysis to check for publication bias. To examine the robustness of the findings we performed two sensitivity analyses, by limiting the included studies to those using operational diagnostic criteria for major depression and to those in which the dosage was $75 \mathrm{mg}$ /day or less.

Subgroup analyses should be performed and interpreted with caution because multiple analyses lead to false positive conclusions. We did, however, perform two subgroup analyses, where possible: for older people (age 65 or more) separately, because these people may be more vulnerable to side effects associated with tricylics and a decreased dosage is often recommended for them; and for psychiatric patients and primary care patients separately, because it is sometimes believed that results obtained from either of these settings may not be straightforwardly applicable to the other setting.

\section{Results}

Study inclusion and characteristics

Of the 2418 citations originally identified in our electronic search, 141 were potentially relevant and

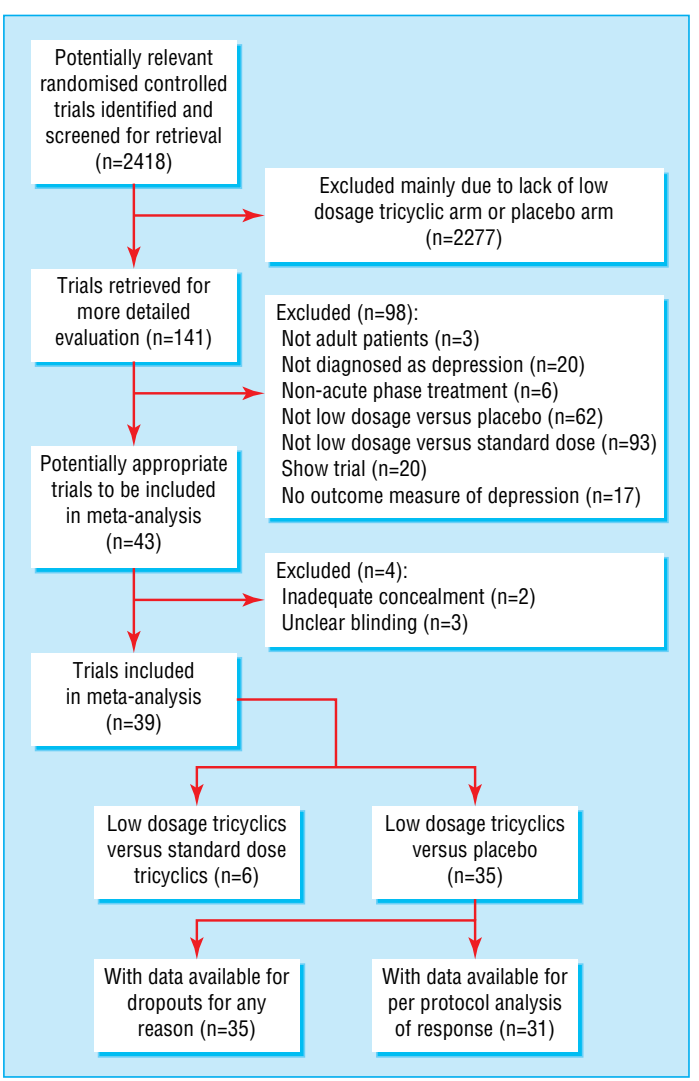

Fig 1 Process of inclusion of studies for review and analysis

were assessed for strict eligibility and quality. The interrater reliability of the two reviewers for this first stage of study selection was good (agreement 97\%, $\kappa=0.61$ ). After the reference search, SciSearch, and personal contacts, we ultimately agreed on 35 studies (2013 participants) that compared low dosage tricyclics with placebo, and six studies (551 participants) that compared low dosage tricyclics with standard dosage tricyclics. Two of these had three arms of standard dosage tricylics, low dosage tricylics, and placebo (fig 1). The inter-rater reliability for this second stage of assessment for eligibility and validity was excellent, with weighted kappas between 0.58 and 0.86 . The inter-rater reliability of the two validity criteria was also satisfactory, with weighted kappas of 0.58 and 0.79 , respectively.

Sixteen studies used amitriptyline as active drugs and 13 used imipramine. The remaining randomised controlled trials studied clomipramine (3 trials), doxepine (3), dothiepin (2), trimipramine (2), and lofepramine (1). Five studies focused on depression in people aged 65 or more. Ten studies were conducted in primary care and 12 studies in psychiatric settings. Six studies dealt with depression seen in patients with comorbid physical conditions such as migraine or rheumatoid arthritis. All of the included studies were randomised controlled trials with both patients and doctors blinded (table; the complete list of included studies is also available in the Cochrane Library). However, only four studies reported enough details on their randomisation procedure. 
Details of included studies

\begin{tabular}{ll} 
Study & $\begin{array}{l}\text { Methods (allocation, blindness, and } \\
\text { duration) }\end{array}$ \\
\hline Ahmed $^{\text {w1 }}$ & Random, "double blind," 12 weeks \\
\hline Blashki $^{\mathrm{w} 2}$ & $\begin{array}{l}\text { Random with adequate concealment, } \\
\text { "double blind," } 4 \text { weeks }\end{array}$ \\
\hline Brick $^{\mathrm{w} 3}$ & Random, "double blind," 7 weeks
\end{tabular}

Participants (diagnosis, age group, patient status)

"Internal heat" $(75 \%$ were potentially depressed), mainly adult, outpatients

Ad hoc operational criteria for depression, mainly adult (mean 38) outpatients at general practice

Mild to severe (except very severe) depression or anxiety according to the Minnesota multiphasic personality inventory and Taylor, mainly adult (mean 34-36), inmates

\begin{tabular}{|c|c|c|c|c|}
\hline Burch $^{\text {w4 }}$ & Random, "double blind," 4-6 weeks & $\begin{array}{l}\text { Primary depressive illness according to } \\
\text { Feighner criteria, mainly adult (range } \\
\text { 18-65), inpatients }\end{array}$ & $\begin{array}{l}\text { Amitriptyline } 40 \mathrm{mg} / \mathrm{d}(28-70 \mathrm{mg} / \mathrm{d}) \text { or } \\
158 \mathrm{mg} / \mathrm{d}(55-280 \mathrm{mg} / \mathrm{d})\end{array}$ & $\begin{array}{l}\text { Montogomery-Asberg depression rating } \\
\text { scale; } \leqslant 9\end{array}$ \\
\hline Burchw5 $^{\text {w5 }}$ & Random, "double blind," 4-6 weeks & $\begin{array}{l}\text { Primary depressive illness according to } \\
\text { Feighner criteria, mainly old (range } \\
\leqslant 65 \text { ), inpatients }\end{array}$ & $\begin{array}{l}\text { Amitriptyline } 57.5 \mathrm{mg} / \mathrm{d}(20-125 \mathrm{mg} / \mathrm{d}) \\
\text { or } 144 \mathrm{mg} / \mathrm{d}(100-190 \mathrm{mg} / \mathrm{d})\end{array}$ & $\begin{array}{l}\text { Montogomery-Asberg depression rating } \\
\text { scale; }<9\end{array}$ \\
\hline Couch $^{\text {w6 }}$ & Random, "double blind," 4 weeks & $\begin{array}{l}\text { Migraine with Hamilton rating scale for } \\
\text { depression- } 18 \geqslant 14 \text {, at least mildly } \\
\text { depressed, mainly adult (range 15-60), } \\
\text { outpatients at headache clinic }\end{array}$ & $\begin{array}{l}\text { Amitriptyline } 94 \mathrm{mg} / \mathrm{d}(50-100 \mathrm{mg} / \mathrm{d}) \text { or } \\
\text { placebo }\end{array}$ & HRSD-18; becoming "non-depressed" \\
\hline Diamond $^{\mathrm{w} 7}$ & Random, "double blind," 4 weeks & $\begin{array}{l}\text { Chronic tension headache with } \\
\text { "depression" or "anxiety and } \\
\text { depression," mainly adult (range 20-60) }\end{array}$ & Amitriptyline $<60 \mathrm{mg} / \mathrm{d}$ or placebo & $\begin{array}{l}4 \text { point scale; excellent or good } \\
\text { according to physician's global } \\
\text { evaluation }\end{array}$ \\
\hline Gram W8 $^{\text {W }}$ & Random, "double blind," 6 weeks & $\begin{array}{l}\text { DSM-III-R major depression, mainly } \\
\text { adult (range 18-70), outpatients and } \\
\text { inpatients }\end{array}$ & $\begin{array}{l}\text { Clomipramine } 25 \mathrm{mg} / \mathrm{d}, 50 \mathrm{mg} / \mathrm{d} \text {, } \\
75 \mathrm{mg} / \mathrm{d}, 125 \mathrm{mg} / \mathrm{d} \text {, or } 200 \mathrm{mg} / \mathrm{d}\end{array}$ & $\begin{array}{l}\text { Hamilton rating scale for depression-17; } \\
\leqslant 7\end{array}$ \\
\hline Fryer"w9 & Random, "double blind," 4 weeks & $\begin{array}{l}\geqslant 70 \text { of T score for depression subscale } \\
\text { of Minnesota multiphasic personality } \\
\text { inventory, mainly adult, inpatients }\end{array}$ & Imipramine $100 \mathrm{mg} / \mathrm{d}$ or placebo & $\begin{array}{l}\text { Minnesota multiphasic personality } \\
\text { inventory depression subscale }\end{array}$ \\
\hline Goldberg $^{\text {w12 }}$ & Random, "double blind," 4 weeks & $\begin{array}{l}\text { "Psychoneurotic (mixed anxiety } \\
\text { depressive), mainly adult (range 19-60), } \\
\text { outpatients }\end{array}$ & $\begin{array}{l}\text { Doxepin } 94.6 \mathrm{mg} / \mathrm{d}(50-100 \mathrm{mg} / \mathrm{d}) \text { or } \\
\text { placebo }\end{array}$ & $\begin{array}{l}\text { Noticeable to moderate change on } \\
\text { overall global improvement }\end{array}$ \\
\hline Goldbergw13 $^{\text {w13 }}$ & Random, "double blind," 6 weeks & $\begin{array}{l}\text { Neurotic depression according to New } \\
\text { York University criteria }\end{array}$ & $\begin{array}{l}\text { Amitriptyline } 91.5 \mathrm{mg} / \mathrm{d}(75-200 \mathrm{mg} / \mathrm{d}) \text {, } \\
\text { placebo, or trazodone }\end{array}$ & $\begin{array}{l}\text { Hamilton rating scale for depression-21; } \\
\geqslant 50 \% \text { reduction in score }\end{array}$ \\
\hline Hollandaw14 & Random, "double blind," 4 weeks & $\begin{array}{l}\text { Endogenous or involutional or reactive } \\
\text { depression according to traditional } \\
\text { criteria, mainly adult (range 17-58), } \\
\text { status not specified }\end{array}$ & Doxepin $60 \mathrm{mg} / \mathrm{d}$ or placebo & $\begin{array}{l}\text { Noticeable to moderate overall } \\
\text { improvement }\end{array}$ \\
\hline Hormazabalw15 $^{\text {w15 }}$ & Random, "double blind," 4 weeks & $\begin{array}{l}\text { Depressive psychosis, depressive } \\
\text { neurosis, reactive depression, and } \\
\text { others, mainly adult (mean 44-42), } \\
\text { mainly outpatients }\end{array}$ & $\begin{array}{l}\text { Amitriptyline } 86.4(\mathrm{SD}=21) \mathrm{mg} / \mathrm{d} \text {, } \\
\text { placebo, or cianopramine }\end{array}$ & $\begin{array}{l}\text { Hamilton rating scale for depression-21; } \\
\text { noticeable to moderate improvement on } \\
\text { global evaluation }\end{array}$ \\
\hline Houstonw16 & Random, "double blind," 52 weeks & $\begin{array}{l}\geqslant 7 \text { on Leeds scale, mainly adult (range } \\
20-46 \text { ), outpatients at general practice }\end{array}$ & $\begin{array}{l}\text { Amitriptyline (slow release) } 50 \mathrm{mg} / \mathrm{d} \text { or } \\
\text { placebo }\end{array}$ & $\begin{array}{l}\text { Leeds scale } D \text { score; } \geqslant 50 \% \text { reduction } \\
\text { in Leeds scale calculated from mean } \\
\text { and SD }\end{array}$ \\
\hline Jacobson w17 & Random, "double blind," 4 weeks & $\begin{array}{l}\text { Depression according to Feighner } \\
\text { criteria, unknown, unknown }\end{array}$ & $\begin{array}{l}\text { Amitriptyline } 75-100 \mathrm{mg} / \mathrm{d} \text {, placebo, or } \\
\text { amitriptyline with chlordiazepoxide }\end{array}$ & $\begin{array}{l}\text { Hamilton rating scale for depression- } 24 \text {; } \\
\geqslant 50 \% \text { reduction in score calculated } \\
\text { from mean and SD }\end{array}$ \\
\hline Jenkins $^{\text {w18 }}$ & Random, "double blind," 4 weeks & $\begin{array}{l}\text { Low back pain with Becks depression } \\
\text { inventory score } \geqslant 14 \text {, mainly adult } \\
\text { (range 18-49), unknown }\end{array}$ & Imipramine $75 \mathrm{mg} / \mathrm{d}$ or placebo & $\begin{array}{l}\text { Beck depression inventory; } \geqslant 50 \% \\
\text { reduction in score }\end{array}$ \\
\hline Kerrw19 & Random, "double blind," 4 weeks & $\begin{array}{l}\text { "Anxiety and depression associated with } \\
\text { menopause," mainly adult (range } \\
\text { 44-57), unknown }\end{array}$ & $\begin{array}{l}\text { Amitriptyline } 55 \mathrm{mg} / \mathrm{d}(30-100 \mathrm{mg} / \mathrm{d}) \text { or } \\
\text { placebo }\end{array}$ & $\begin{array}{l}\text { One item assessment in } 5 \text { grades; } \\
\text { "marked improvement or complete } \\
\text { relief of symptoms" }\end{array}$ \\
\hline Laederach-Hofmann ${ }^{\text {w20 }}$ & Random, "double blind," 8 weeks & $\begin{array}{l}\text { Obese binge eaters not being anorexic } \\
\text { or bulimic according to DSM-IV } \\
\text { (baseline Hamilton rating scale for } \\
\text { depression-21 scores were } 21 \text { to } 23 \text { on } \\
\text { average), mainly adult (range 20-60), } \\
\text { outpatients }\end{array}$ & Imipramine $75 \mathrm{mg} / \mathrm{d}$ or placebo & $\begin{array}{l}\text { Hamilton rating scale for depression-21, } \\
\text { modified; } \geqslant 50 \% \text { reduction in score } \\
\text { calculated from mean and SD }\end{array}$ \\
\hline Lecrubierw21 w22 $^{\text {wh }}$ & Random, "double blind," 6 months & $\begin{array}{l}\text { DSM-III-R dysthymia (40\%), dysthymia } \\
\text { with major depression }(40 \%) \text {, and } \\
\text { major depression in partial remission } \\
(20 \%) \text {; mainly adult (range } 18-73 \text {, } \\
\text { mean } 43) \text {; outpatients }\end{array}$ & $\begin{array}{l}\text { Imipramine } 100 \mathrm{mg} / \mathrm{d} \text {, placebo, or } \\
\text { amisulpiride }\end{array}$ & $\begin{array}{l}\text { Montgomery-Asberg depression rating } \\
\text { scale; much improved or much } \\
\text { improved on clinical global impression }\end{array}$ \\
\hline Macfarlane ${ }^{\text {w23 }}$ & Random, "double blind," 12 weeks & $\begin{array}{l}\text { Rheumatoid arthritis with score of } \geqslant 50 \\
\text { on Zung self rating depression scale, } \\
\text { mainly adult (range 18-73), outpatients }\end{array}$ & Trimipramine $25-75 \mathrm{mg} / \mathrm{d}$ or placebo & $\begin{array}{l}\text { Zung self rating depression scale; } \\
\geqslant 50 \% \text { reduction in score calculated } \\
\text { from mean and SD }\end{array}$ \\
\hline Morakinyow24 & Random, "double blind," 6 weeks & $\begin{array}{l}\text { "Depression" according to traditional } \\
\text { diagnosis, mainly adult (range 20-50), } \\
\text { unknown }\end{array}$ & $\begin{array}{l}\text { Amitriptyline } 75 \mathrm{mg} / \mathrm{d} \text {, placebo, or } \\
\text { amitriptyline with chlordiazepoxide }\end{array}$ & $\begin{array}{l}\text { "Improved" according to overall } \\
\text { response in three grades of "improved," } \\
\text { "doubtful," and "not improved" }\end{array}$ \\
\hline Murphy ${ }^{\text {w25 w26 }}$ & Random, "double blind," 6 weeks & $\begin{array}{l}\text { "Depression" according to traditional } \\
\text { diagnosis, mainly adult (range 18-70), } \\
\text { outpatients at general practice }\end{array}$ & $\begin{array}{l}\text { Imipramine } 100 \mathrm{mg} / \mathrm{d} \text {, placebo, or } \\
\text { mianserin }\end{array}$ & $\begin{array}{l}\text { Ad hoc physician scale; } \geqslant 50 \% \\
\text { reduction in score calculated from } \\
\text { mean and SD }\end{array}$ \\
\hline
\end{tabular}

Interventions

Outcomes (depression severity and response) benzoctamin

Amitriptyline $150 \mathrm{mg} / \mathrm{d}$ or $75 \mathrm{mg} / \mathrm{d}$ or Hamilton rating scale for depression-17; placebo

Amitriptyline $30 \mathrm{mg} / \mathrm{d}$, placebo, or $\geqslant 50 \%$ reduction in score calculated from mean and SD

Minnesota multiphasic personality inventory-depression scale; excellent or good response according to patients subjective evaluation

amitriptyline with emylcamate$$
\text { . }
$$ 
Details of included studies contd

Participants (diagnosis, age group,

\begin{tabular}{ll} 
Study & $\begin{array}{l}\text { Methods (allocation, blindness, and } \\
\text { duration) }\end{array}$ \\
\hline Nandiw27 & $\begin{array}{l}\text { Random with adequate allocation } \\
\text { concealment, "double blind," } 4 \text { weeks }\end{array}$ \\
\hline Petraca $^{\text {w28 }}$ & Random, "double blind," 6 weeks \\
\hline Philipp $^{\text {w29 }}$ & Random, "double blind," 8 weeks
\end{tabular}
patient status)

"Depression" according to traditional diagnosis, mainly adult, community residents

DSM-III-R dysthymia or major depression, mainly old (mean 72), unknown

\section{CD-10 moderate depressive episode} (patients with mild or severe condition excluded), mainly adult (range 18-65), outpatients at general practice

\begin{tabular}{|c|c|}
\hline Rampellow30 & Random, "double blind," 6 weeks \\
\hline Reiflerw31 w32 & Random, "double blind," 8 weeks \\
\hline Rickelsw33 w34 & Random, "double blind," 4 weeks \\
\hline Rickels $^{\text {w35 }}$ & Random, "double blind," 4 weeks \\
\hline Robertson ${ }^{w 36}$ & Random, "double blind," 6 weeks \\
\hline Rouillon"w37 & Random, "double blind," 8 weeks \\
\hline Schweizer $^{v 38 \text { w }}$ & Random, "double blind," 8 weeks \\
\hline
\end{tabular}

DSM-III-R major depression or bipolar depression, anxious, mainly adult (range 18-62), outpatients

Alzheimer's disease with major depression (DSM-III), mainly old (mean 72), outpatients "Mildly to moderately depressed"
(reactive neurotic depression, mixed anxiety depressive reaction), mainly adult (mean 43-45), outpatients

"Symptoms of depression and anxiety," Amitriptyline $70 \mathrm{mg} / \mathrm{d}(50-100 \mathrm{mg} / \mathrm{d})$ or Physician depression scale; moderate to mainly adult, volunteers

placebo

Interventions

Imipramine $97.4 \mathrm{mg} / \mathrm{d}$, placebo, or natural process

Clomipramine 100 mg/d or placebo

mipramine $100 \mathrm{mg} / \mathrm{d}$, placebo or

hypericum extract

Amitriptyline $100 \mathrm{mg} / \mathrm{d}$, placebo, or amineptine

Imipramine $83 \mathrm{mg} / \mathrm{d}$ or placebo

Amitriptyline $100 \mathrm{mg} / \mathrm{d}$ or placebo

Esith research diagnostic criteria major depression, mainly adult (range 18-70), unknown

DSM-III-R residual depression, mainly adult (range 18-65), outpatients

DSM-III-R unipolar major depression, Imipramine 89 mg/d (25-150 mg/d) or comorbid with various chronic physical placebo conditions, mainly old (range 65-89, mean 72), outpatients

\begin{tabular}{|c|c|c|c|c|}
\hline Simpson ${ }^{w 40}$ & Random, "double blind," 6 weeks & $\begin{array}{l}\text { Research diagnostic criteria } \\
\text { endogenous major depression, mainly } \\
\text { adult (range 22-60), outpatients }\end{array}$ & Trimipramine $75 \mathrm{mg} / \mathrm{d}$ or $150 \mathrm{mg} / \mathrm{d}$ & $\begin{array}{l}\text { Hamilton rating scale for depression- } 21 \\
\text { noticeable to moderate improvement on } \\
\text { clinical global impression }\end{array}$ \\
\hline $\operatorname{Tan}^{1 / 41}$ w42 & Random, "double blind," 4 weeks & $\begin{array}{l}\text { "Depression" without dementia or } \\
\text { major physical illness, mainly old ( }>65 \text {, } \\
\text { mean 80), inpatients }\end{array}$ & Lofepramine $70 \mathrm{mg} / \mathrm{d}$ or placebo & $\begin{array}{l}\text { Montgomery-Asberg depression rating } \\
\text { scale; } \geqslant 50 \% \text { reduction in score } \\
\text { calculated from mean and SD }\end{array}$ \\
\hline Tetreault ${ }^{\mathrm{w} 43}$ & Random, "double blind," 6 weeks & $\begin{array}{l}\text { Kiloh and Garside's "neurotic reactive } \\
\text { depression," not specified, inpatients }\end{array}$ & Imipramine $50-100 \mathrm{mg} / \mathrm{d}$ or placebo & $\begin{array}{l}\text { Wechsler scale; } \geqslant 50 \% \text { reduction in } \\
\text { score calculated from mean and SD }\end{array}$ \\
\hline Thompson ${ }^{\text {w44 }}$ & Random, "double blind," 4 weeks & $\begin{array}{l}\text { Doctor's usual diagnosis of } \\
\text { "depression" ( } 73 \% \text { were research } \\
\text { diagnostic criteria definite or probable } \\
\text { major depression), not specified, } \\
\text { outpatients at general practice }\end{array}$ & Dothiepin $75 \mathrm{mg} / \mathrm{d}$ or placebo & $\begin{array}{l}\text { Hamilton rating scale for depression-17; } \\
\geqslant 50 \% \text { reduction in score calculated } \\
\text { from mean and SD }\end{array}$ \\
\hline Tyrer"145 w46 & Random, "double blind," 6 weeks & $\begin{array}{l}\text { DSM-III dysthymic disorder, mainly } \\
\text { adult (range 17-76), outpatients at } \\
\text { general practice }\end{array}$ & $\begin{array}{l}\text { Dothiepin } 51.3 \mathrm{mg} / \mathrm{d}(25-150 \mathrm{mg} / \mathrm{d}) \text { or } \\
\text { placebo }\end{array}$ & $\begin{array}{l}\text { Montgomery-Asberg depression rating } \\
\text { scale }\end{array}$ \\
\hline Weissman ${ }^{\text {w47 }}$ & Random, "double blind," 6 weeks & $\begin{array}{l}\text { DSM-III moderate to severe major } \\
\text { depression, with minor chronic physical } \\
\text { conditions, mainly old (range 60-85), } \\
\text { outpatients }\end{array}$ & $\begin{array}{l}\text { Imipramine } 97.5 \mathrm{mg} / \mathrm{d}(25-225 \mathrm{mg} / \mathrm{d}) \\
\text { or placebo }\end{array}$ & $\begin{array}{l}\text { Hamilton rating scale for depression-23; } \\
\geqslant 50 \% \text { reduction in score calculated } \\
\text { from mean and SD }\end{array}$ \\
\hline WHO (Lucknow) ${ }^{\mathrm{w} 49}$ & Random, "double blind," 4 weeks & $\begin{array}{l}\text { ICD-9 manic depressive illness or } \\
\text { neurotic depression, mainly adult } \\
\text { (range 18-65), outpatients }(20 \%) \text { and } \\
\text { inpatients }(80 \%)\end{array}$ & $\begin{array}{l}\text { Imipramine } 37.5-75 \mathrm{mg} / \mathrm{d} \text { or } 75-150 \\
\mathrm{mg} / \mathrm{d}\end{array}$ & $\begin{array}{l}\text { Hamilton rating scale for depression-17; } \\
\geqslant 50 \% \text { reduction in score calculated } \\
\text { from mean and SD }\end{array}$ \\
\hline WHO (Nagasaki) ${ }^{\text {w50 }}$ & Random, "double blind," 4 weeks & $\begin{array}{l}\text { ICD-9 manic depressive illness or } \\
\text { neurotic depression, mainly adult } \\
\text { (range 18-65), outpatients }(85 \%) \text { and } \\
\text { inpatients }(15 \%)\end{array}$ & $\begin{array}{l}\text { Amitriptyline } 37.5-75 \mathrm{mg} / \mathrm{d} \text { or } 75-150 \\
\mathrm{mg} / \mathrm{d}\end{array}$ & $\begin{array}{l}\text { Hamilton rating scale for depression-17; } \\
\geqslant 50 \% \text { reduction in score calculated } \\
\text { from mean and SD }\end{array}$ \\
\hline WHO (Nashville)"w51 & Random, "double blind," 4 weeks & $\begin{array}{l}\text { ICD-9 manic depressive illness or } \\
\text { neurotic depression, mainly adult } \\
\text { (range 18-65), outpatients }\end{array}$ & $\begin{array}{l}\text { Imipramine } 37.5-75 \mathrm{mg} / \mathrm{d} \text { or } 75-150 \\
\mathrm{mg} / \mathrm{d}\end{array}$ & $\begin{array}{l}\text { Hamilton rating scale for depression-17; } \\
\geqslant 50 \% \text { reduction in score calculated } \\
\text { from mean and SD }\end{array}$ \\
\hline
\end{tabular}

w1 Ahmed MH, Onyemelukwe GC, Onyewoto II. A double blind controlled clinical trial of benzoctamine (Tacitin) and imipramine (Tofranil) in the treatment of "internal heat" and its associated symptoms. East African Medical Journal 1988:65(4):230-7.

w2 Blashki TG, Mowbry R, Davies B. Controlled trial of amitriptyline in general practice. British Medical Journal 1971:1:133-8.

W3 Brick H, Doub WH- Jr, Perdue WC. Effects of amitriptyline on depressive and anxiety states in penitentiary inmates. Diseases of the Nervous System 1962;23:572-8.

w4 Burch JE, Ahmed 0, Hullin RP, Mindham RH. Antidepressive effect of amitriptyline treatment with plasma drug levels controlled within three different ranges. Psychopharmacology 1988;94(2):197-205.

w5 Burch JE, Ahmed 0, Hullin RP, Mindham RH. Antidepressive effect of amitriptyline treatment with plasma drug levels controlled within three different ranges. Psychopharmacology 1988;94(2):197-205

w6 Couch JR, Hassanein RS. Amitriptyline in migraine prophylaxis. Archives of Neurology 1979;36(11):695-9

w7 Diamond S, Baltes BJ. Chronic tension headache - treated with amitriptyline - a double-blind study. Headache 1971;11(3):110-6.

w8 Gram LF, Kragh-Sorensen P, Bech P, Bolwig TG, Vestergaard P, Larsen JK. Clomipramine dose-effect study in patients with depression: clinical end points and pharmacokinetics. Clinical

Pharmacology and Therapeutics 1999;66(2):152-65.

w9 Fryer DG, Timberlake WD. A trial of imipramine (Tofranil) in depressed patients with chronic physical disease. Journal of Chronic Diseases 1963;16:173-8. 
Details of included studies contd

w10 Goldberg HL, Finnery RJ. The use of doxepin in the treatment of symptoms of anxiety neurosis and accompanying depression: a collaborative controlled study. American Journal of Psychiatry 1972;129(1):74-7.

w11 Goldberg HL, Finnerty RJ, Cole J0. Doxepin: is a single daily dose enough? American Journal of Psychiatry 1974;131(9):1027-9.

w12 Goldberg HL, Finnerty RJ. Trazodone in the treatment of neurotic depression. Journal of Clinical Psychiatry 1980;41(12Pt1):430-4.

w13 Goldberg HL, Finnerty RJ, Cole J0. Doxepin: is a single daily dose enough? American Journal of Psychiatry 1974;131(9):1027-9.

w14 Hollanda Junior L, Silva CN, da, Lira BS, Ferreira SC. Double-blind clinical study with a new psychotropic agent: doxepin versus placebo. Hospital Rio Journal 1970;77(3):799-803.

w15 Hormazabal L, Omer LM, Ismail S. Cianopramine and amitriptyline in the treatment of depressed patients: a placebo-controlled study. Psychopharmacology 1985;86(1-2):205-8.

w16 Houston J, Berg I, Butler A, McGuire R. Amitriptyline for depressed women with young children in general practice. British Journal of Psychiatry 1983;142:103-4.

w17 Jacobson AF. Doctor-patient concordance in a placebo-controlled trial of limbitrol versus its components proceedings. Psychopharmacology Bulletin 1978;14(3):61-3.

w18 Jenkins DG, Ebbutt AF, Evans CD. Tofranil in the treatment of low back pain. Journal of International Medical Research 1976;4:28-40.

w19 Kerr MM. Amitriptyline in emotional states at the menopause. New Zealand Medical Journal 1970;72(461):243-5.

w20 Laederach-Hofmann K, Graf C, Horber F, Lippuner K, Lederer S, Michel R, Schneider M. Imipramine and diet counseling with psychological support in the treatment of obese binge eaters: a randomized, placebo-controlled double-blind study. International Journal of Eating Disorders 1999;26(3):231-44.

w21 Boyer P, Lecrubier Y. Atypical antipsychotic drugs in dysthymia: placebo controlled studies of amisulpride versus imipramine, versus amineptine. European Psychiatry: the Journal of the Association of European Psychiatrists 1996;11(suppl 3):S135-40.

w22 Lecrubier Y, Boyer P, Turjanski S, Rein W. Amisulpride versus imipramine and placebo in dysthymia and major depression Amisulpride Study Group. Journal of Affective Disorders 1997;43(2):95-103

w23 Macfarlane JG, Jalali S, Grace EM. Trimipramine in rheumatoid arthritis: a randomized double-blind trial in relieving pain and joint tenderness. Current Medical Research and Opinion 1986;10:89-93.

w24 Morakinyo VO. Amytriptyline and chlordiazepoxide (Limbitrol) in depressive states in Nigeriansa double-blind study. African Journal of Medical Sciences 1970;1(4):409-14. w25 Murphy JE, Donald JF, Molla AL. Mianserin in the treatment of depression in general-practice. Practitioner 1976;217(1297):135-8.

w26 Murphy JE. Mianserin in the treatment of depressive illness and anxiety states in general-practice. British Journal of Clinical Pharmacology 1978;5(suppl 1):81S-5S.

w27 Nandi DN, Ajmany S, Ganguli H, Banerjee G, Boral GC, Ghosh A, Sarkar S. A clinical evaluation of depressives found in a rural survey in India. British Journal of Psychiatry 1976;128:523-7. w28 Petracca G, Teson A, Chemerinski E, Leiguarda R, Starkstein SE. A double-blind placebo-controlled study of clomipramine in depressed patients with Alzheimer's disease. Journal of Neuropsychiatry and Clinical Neurosciences 1996;8(3):270-5.

w29 Philipp M, Kohnen R, Hiller K-0. Hypericum extract versus imipramine or placebo in patients with moderate depression: randomised multicentre study of treatment for eight weeks. British Medical Journal 1999;319:1534-8.

w30 Rampello L, Nicoletti G, Raffaele R, Drago F. Comparative effects of amitriptyline and amineptine in patients affected by anxious depression. Neuropsychobiology 1995;31(3):130-4. w31 Reifler BV, Teri L, Raskind M, Veith R, Barnes R, White E, McLean P. Double-blind trial of imipramine in Alzheimer's disease patients with and without depression. American Journal of Psychiatry 1989;146(1):45-9.

w32 Teri L, Reifler BV, Veith RC, Barnes R, White E, McLean P, Raskind M. Imipramine in the treatment of depressed Alzheimer's patients: impact on cognition. Journal of Gerontology 1991;46(6):P372-7.

w33 Rickels K, Gordon PE, Jenkins BW, Perloff M, Sachs T, Stepansky W. Drug treatment in depressive illness. Diseases of the Nervous System 1970;31(1):30-42. w34 Rickels K, Hesbacher P, Downing RW. Differential drug effects in neurotic depression. Diseases of the Nervous System 1970;31(7):468-75.

w35 Rickels K, Csanalosi I, Chung HR, Case WG, Pereira Ogan A, Downing RW. Amitriptyline in anxious-depressed outpatients: a controlled study. American Journal of Psychiatry 1974;131(1):25-30

w36 Robertson MM, Trimble MR. The treatment of depression in patients with epilepsy A double-blind trial. Journal of Affective Disorders 1985;9(2):127-36

w37 Rouillon F, Markabi S, Febvre N, Phillips R, Vaillant J. Controlled study of treatment of residual depression by clomipramine versus placebo. Encephale 1994;20(2):139-45.

w38 Schweizer E, Rickels K. Hassman H. A double-blind, placebo-controlled comparison of imipramine and buspirone in the treatment of major depression in the elderly in the community. Psychopharmacology Bulletin 1994:639.

w39 Schweizer E, Rickels K, Hassman H, Garcia Espana F. Buspirone and imipramine for the treatment of major depression in the elderly. Journal of Clinical Psychiatry 1998;59(4):175-83. w40 Simpson GM, Pi EH, Gross L, Baron D, November M. Plasma levels and therapeutic response with trimipramine treatment of endogenous depression. Journal of Clinical Psychiatry 1988;49(3):113-6.

w41 Tan RS, Barlow RJ, Abel C, Reddy S, Palmer AJ, Fletcher AE, Nicholl CG, Pitt BM, Bulpitt CJ. The effect of low dose lofepramine in depressed elderly patients in general medical wards. British Journal of Clinical Pharmacology 1994;37(4):321-4.

w42 Tan RS. Lowering antidepressant dosages in the elderly. Clinical Gerontologist 1995;16(1):67-70.

w43 Tetreault L, Doucet P, Blanchet A, Bordeleau JM. Comparative evaluation of the antidepressive properties of opipramol, imipramine and placebo in neurotic depression. L'union Médicale du Canada 1966;95(5):546-53.

w44 Thompson C, Thompson CM. The prescribing of antidepressants in general practice II: a placebo-controlled trial of low-dose dothiepin. Human Psychopharmacology 1989:4:191-204. w45 Tyrer P, Seivewright N, Murphy S, Ferguson B, Kingdon D, Barczak P, Brothwell J, Darling C, Gregory S, Johnson AL. The Nottingham study of neurotic disorder: comparison of drug and psychological treatments. Lancet 1988;2:235-40.

w46 Tyrer P, Seivewright N, Ferguson B, Murphy S, Darling C, Brothwell J, Kingdon D, Johnson AL. The Nottingham Study of Neurotic Disorder: relationship between personality status and symptoms. Psychological Medicine 1990;20(2):423-31.

w47 Weissman MM, Prusoff B, Sholomskas AJ, Greenwald S. A double-blind clinical trial of alprazolam, imipramine, or placebo in the depressed elderly. Journal of Clinical Psychopharmacology 1992;12(3):175-82.

w48 Dose effects of antidepressant medication in different populations A World Health Organization collaborative study. Journal of Affective Disorders 1986;(suppl 2):1-67. w49 Dose effects of antidepressant medication in different populations A World Health Organization collaborative study. Journal of Affective Disorders 1986;(suppl 2):1-67. w50 Dose effects of antidepressant medication in different populations A World Health Organization collaborative study. Journal of Affective Disorders 1986;(suppl 2):1-67. w51 Dose effects of antidepressant medication in different populations A World Health Organization collaborative study. Journal of Affective Disorders 1986;(suppl 2):1-67.

\section{Low dosage tricyclics versus placebo}

\section{Effectiveness}

Low dosage tricyclics, on average between 75 and 100 $\mathrm{mg} /$ day, were $65 \%(36 \%$ to $100 \%$; random effects model), $47 \%$ (12\% to $94 \%$ ), and $114 \%$ ( $41 \%$ to $226 \%$ ) more likely than placebo to bring about response at 4 weeks, 6-8 weeks, and 3-12 months, respectively. On average $45 \%$ of patients taking low dosage tricyclics responded at 4 weeks, $59 \%$ at $6-8$ weeks, and $53 \%$ at 3-12 months. Heterogeneity was noted only for the outcome at 6-8 weeks (fig 2).

This advantage of low dosage tricyclics was not maintained when we undertook the strict intention to treat analyses based on the worst case scenario. Effectiveness was, however, corroborated by secondary analyses based on continuous measures. People taking low dosage tricyclics had scores for severity of depression that were 0.29 ( 0 to 0.59 ; random effects model), 0.59 ( 0.30 to 0.87$), 0.59$ ( 0.20 to 0.99 ), and 0.89 (0.10 to
1.68) standard deviations lower than those taking placebo at 2 weeks, 4 weeks, 6-8 weeks, and 3-12 months, respectively. Heterogeneity was noted for all these time periods (fig 3).

\section{Acceptability}

No difference was found in total number of dropouts between low dosage tricyclics and placebo groups (relative risk 1.08, 0.93 to 1.26). Overall, 439 of 1840 (24\%) enrolled participants dropped out by the end of the trial. People taking low dosage tricyclics, however, were $111 \%$ (35\% to $228 \%$ ) more likely than those taking placebo to drop out due to side effects. People taking low dosage tricylics were also $63 \%$ (36\% to $95 \%$ ) more likely to experience at least one side effect.

\section{Funnel plot analysis and sensitivity analyses}

The funnel plot showed some publication bias because the five smallest studies reported large relative risks in 


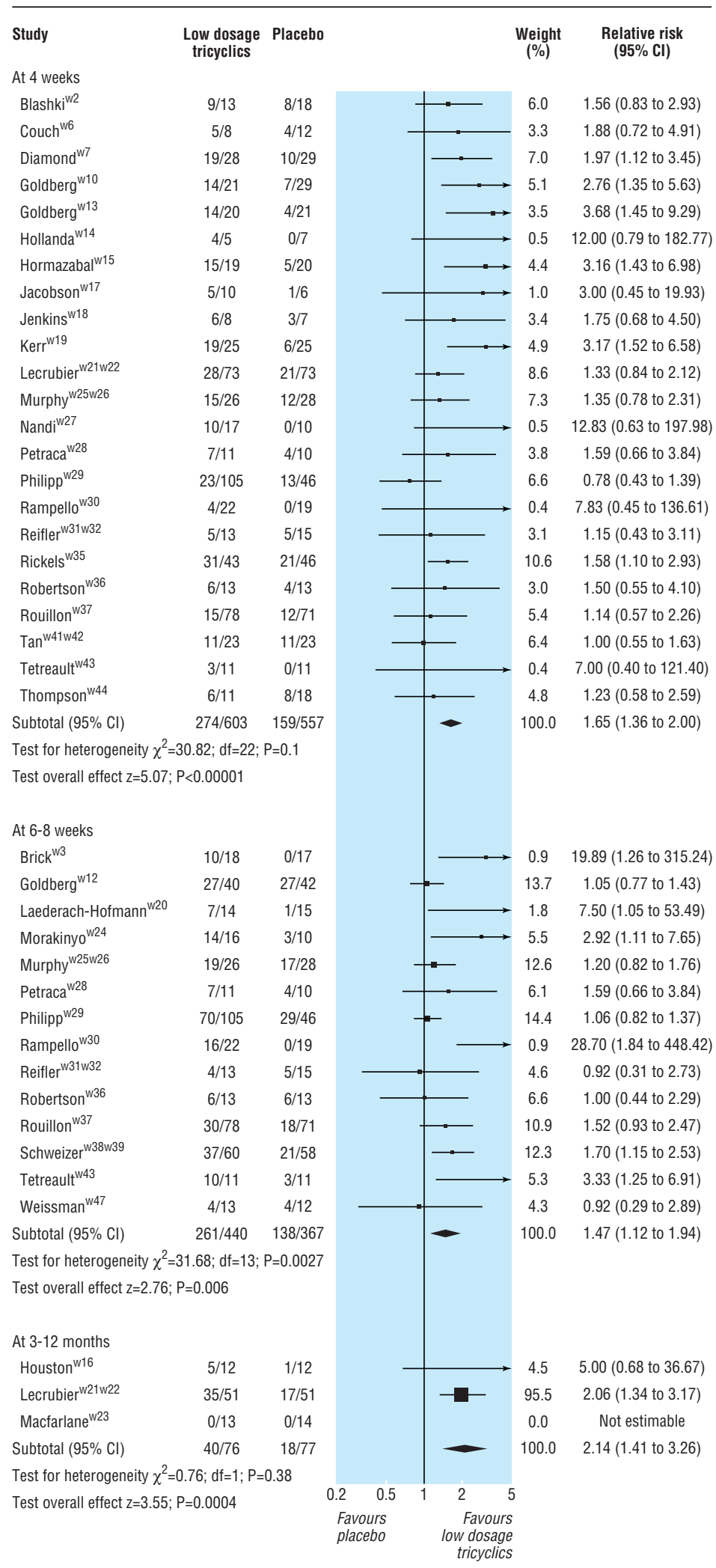

Fig 2 Low dosage tricyclics versus placebo for all depression: per protocol relative risk of response the relative risk decreased only slightly from 1.65 (1.36 to 2.00 ) to 1.58 (1.31 to 1.90 ) at 4 weeks, and from 1.47 (1.12 to 1.94$)$ to 1.28 (1.05 to 1.55$)$ at $6-8$ weeks. The outcome at 6-8 weeks was no longer heterogeneous. The pooled standardised mean difference for the continuous outcome changed to $-0.31(-0.47$ to -0.15$)$ at 4 weeks and $-0.32(-0.49$ to -0.15$)$ at $6-8$ weeks; these results were also no longer heterogeneous.

When we limited the included studies to those that used operational diagnostic criteria for depression, the results were essentially identical. When we limited the included studies to patients taking less than $75 \mathrm{mg}$ /day of tricyclics they were still more likely to show response than those taking placebo at 4 weeks (relative risk 1.63, 1.29 to 2.07 ). The corresponding standardised mean difference in measurements of depression was -0.44 $(-0.72$ to -0.17$)$. Patients receiving this minimal dosage were still more likely to drop out due to side effects (relative risk 2.17, 1.05 to 4.50 ) or to experience at least one side effect (relative risk 2.18, 1.28 to 3.73) than those taking placebo.

\section{Subgroup analyses}

Based on these sensitivity analyses, we did not need to restrict ourselves to studies employing operational diagnostic criteria or to those that administered strictly low dosage tricyclics to arrive at conclusions generalisable to present day patients. The following subgroup analyses therefore deal with studies treating any depression.

Old people-Only five studies explicitly dealt with depression in people aged 65 or more $(n=265)$. Due to lack of power, the meta-analysis of these five studies produced only the following significant findings. The people taking low dosage tricyclics were more likely to show response at 6-8 weeks than those taking placebo, but they were also more likely to experience at least one side effect (relative risk 1.52, 1.09 to 2.11 and 1.26, 1.10 to 1.45 , respectively). The point estimates of the obtained relative risks and standardised mean differences were in accordance with the overall results.

Primary care settings-Five studies recruited patients with depression in primary care settings; five further studies included patients with various physical conditions such as migraine, low back pain, and rheumatoid arthritis, presumably in primary care settings.

Overall there were 558 participants. Although there were no significant findings in the random effects estimates of our primary outcome, the resulting 95\% confidence intervals were compatible with the overall findings. For example, at 4 weeks participants taking low dosage tricyclics were $28 \%$ (-2\% to $68 \%$; random effects model) more likely to show response than those taking placebo; at 6-8 weeks, participants taking low dosage tricyclics were $23 \%$ (-3\% to $55 \%)$ more likely to do so. The continuous outcomes were again supportive of the overall conclusions because the standardised mean difference was $-0.29(-0.58$ to 0.01$)$ at 4 weeks and $-0.41(-0.62$ to -0.19$)$ at $6-8$ weeks.

Psychiatric settings-We also performed a metaanalysis on only such studies that made clear that they were conducted with patients seen in psychiatric settings and not comorbid with other major psychiatric disorders such as eating disorders or Alzheimer's disease. Twelve studies ( $\mathrm{n}=912)$ were available; two with inpatients and the others with outpatients. 


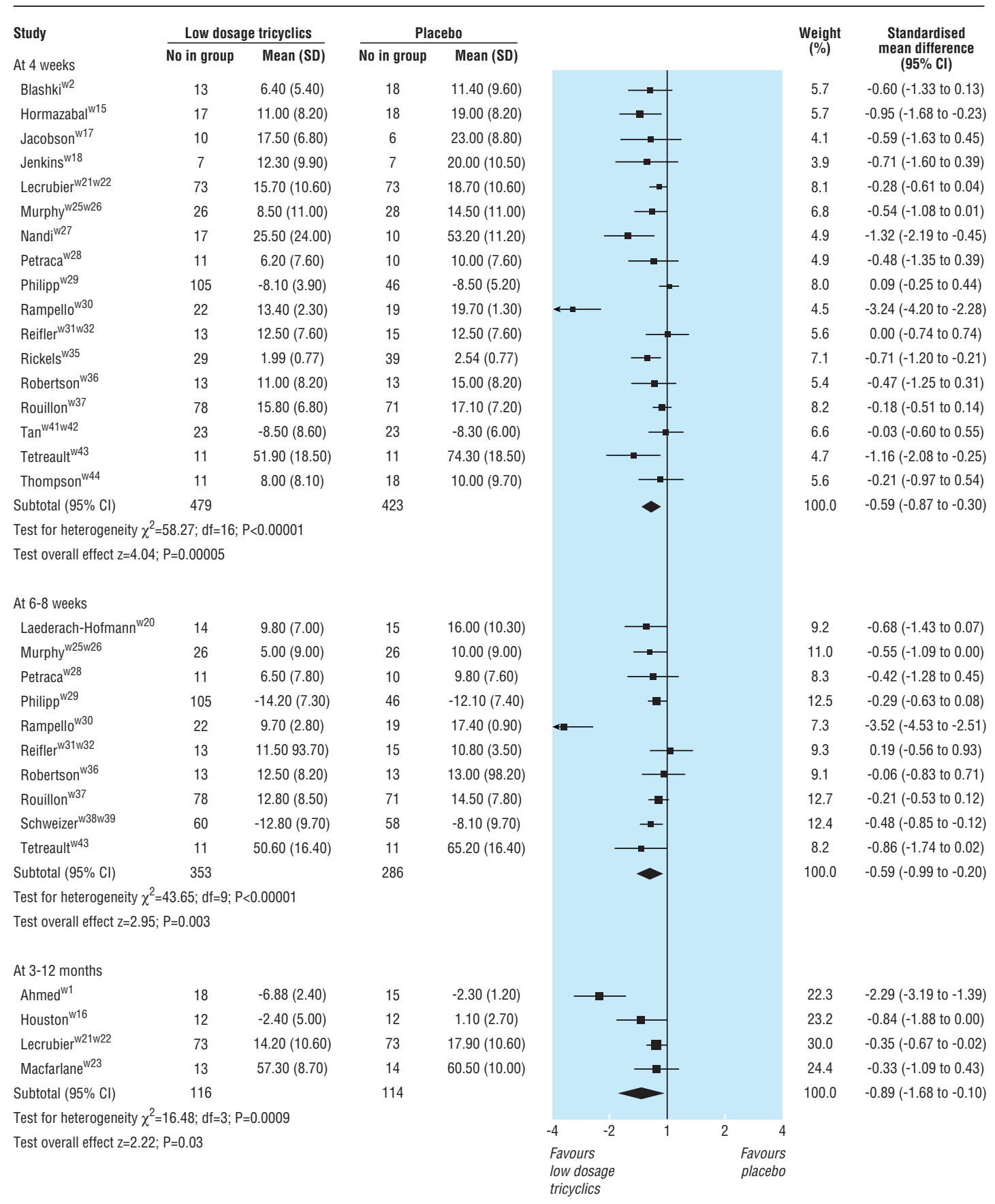

Fig 3 Low dosage tricyclics versus placebo for all depression: per protocol standardised weighted mean difference in depressive severity

Among the psychiatric patients, the relative risk for showing response with low dosage tricyclic rather than with placebo was 2.40 (1.11 to 5.16 ; random effects model) at 2 weeks, 1.89 (1.24 to 2.87$)$ at 4 weeks, 1.66 (0.87 to 3.15$)$ at $6-8$ weeks, and 2.06 (1.34 to 3.17) at 6 months. The standardised mean difference also supported the effectiveness of low dosage tricyclics in psychiatric patients with depression. Exclusion of the outliers lessened heterogeneity associated with some of the results but did not substantially affect the relative risks or standardised mean differences. The tricyclic was more likely to cause dropouts due to side effects or at least one side effect (relative risk 3.80, 1.63 to 8.86 and $1.43,1.19$ to 1.73 , respectively) than placebo.

\section{Low dosage tricyclics versus standard dosage tricyclics}

Effectiveness

Standard dosage tricyclics were not significantly more effective at achieving response than low dosage tricyclics at $1-8$ weeks (fig 4$)$ : relative risk 0.89 ( 0.74 to $1.07)$ at 4 weeks and $1.11(0.76$ to 1.61$)$ at $6-8$ weeks. In terms of the standardised mean difference, standard dosage tricyclics outperformed the low dosage 


\begin{tabular}{|c|c|c|c|c|c|}
\hline Study & $\begin{array}{l}\text { Low dosage } \\
\text { tricyclics } n / N\end{array}$ & $\begin{array}{l}\text { Standard dosage } \\
\text { tricyclics } n / N\end{array}$ & & $\begin{array}{c}\text { Weight } \\
(\%)\end{array}$ & $\begin{array}{c}\text { Relative risk } \\
(95 \% \mathrm{CI})\end{array}$ \\
\hline \multicolumn{6}{|l|}{ At 4 weeks } \\
\hline Blashki $^{\text {w2 }}$ & $9 / 13$ & $11 / 14$ & & 16.1 & 0.88 (0.56 to 1.39$)$ \\
\hline Burch $^{\text {w4 }}$ & $1 / 16$ & $11 / 34$ & & 0.9 & 0.19 (0.03 to 1.37$)$ \\
\hline Gram $^{\text {w8 }}$ & $27 / 70$ & $22 / 42$ & & 19.3 & $0.74(0.49$ to 1.11$)$ \\
\hline WHO(Cali) ${ }^{\text {w48 }}$ & $26 / 33$ & $24 / 31$ & & 45.4 & 1.02 (0.78 to 1.32$)$ \\
\hline WHO(Lucknow) ${ }^{\text {w49 }}$ & $12 / 24$ & $13 / 25$ & & 11.1 & 0.96 (0.56 to 1.67$)$ \\
\hline WHO(Nagasaki) w50 & $5 / 15$ & $7 / 13$ & - & 4.5 & $0.62(0.26$ to 1.48$)$ \\
\hline WHO(Nashville) $)^{\text {w51 }}$ & $4 / 18$ & $5 / 17$ & & 2.7 & 0.76 (0.24 to 2.35$)$ \\
\hline Subtotal $(95 \% \mathrm{Cl})$ & $84 / 189$ & $93 / 176$ & & 100.0 & 0.89 (0.74 to 1.07$)$ \\
\hline \multicolumn{6}{|c|}{ Test for heterogeneity $\chi^{2}=6.19 ; d f=6 ; P=0.4$} \\
\hline \multicolumn{6}{|c|}{ Test overall effect $z=-1.26 ; P=0.2$} \\
\hline \multicolumn{6}{|l|}{ At 6-8 weeks } \\
\hline Burch $^{\text {w4 }}$ & $2 / 16$ & $12 / 34$ & & 6.5 & 0.35 (0.09 to 1.40$)$ \\
\hline Gram8 & $25 / 91$ & $17 / 60$ & & 26.6 & $0.97(0.57$ to 1.64$)$ \\
\hline Diamond ${ }^{\mathrm{w} 7}$ & $19 / 28$ & $16 / 28$ & - & 33.3 & 1.19 (0.79 to 1.79$)$ \\
\hline Simpson ${ }^{w 40}$ & $10 / 10$ & $7 / 10$ & - & 33.6 & 1.43 (0.95 to 2.14$)$ \\
\hline Subtotal $(95 \% \mathrm{Cl})$ & $56 / 145$ & $52 / 132$ & & 100.0 & $1.11(0.76$ to 1.61$)$ \\
\hline \multicolumn{3}{|c|}{ Test for heterogeneity $\chi^{2}=5.70 ; d f=3 ; P=0.13$} & 25 & 5 & \\
\hline \multicolumn{6}{|c|}{ Test overall effect $z=0.54 ; P=0.6$} \\
\hline
\end{tabular}

Fig 4 Low dosage tricyclics versus standard dosage tricyclics for all depression: per protocol relative risk of response not high overall, as our worst case scenario intention to treat analyses showed, they were large enough to hamper drawing definitive conclusions. The dropout is always a problem but here it is even more prominent because, in the case of low dosage tricyclics, there is a trade-off between response and dropouts. If dropouts are not dealt with appropriately, the higher dosage always wins because it increases response at the expense of dropouts. Secondly, the quality of reporting in the included studies was not ideal. We are uncertain whether the random allocation was adequately concealed in most of the studies. Some studies failed to report standard deviations for their outcome measures. Thirdly, and perhaps due to the above factors, we noted heterogeneity for some of the pooled results. A few studies were extreme outliers, all in favour of the low dosage regimen. Lastly, most of the included studies lasted up to eight weeks only.

We evaluated the seriousness of these shortcomings with several sensitivity analyses. Omitting the positive small studies removed heterogeneity of the pooled analyses and yet showed little changes in relative risks and standardised mean differences. Limiting the studies to those that employed modern operational diagnostic criteria or those that used strictly low dosage regimens did not materially affect the pooled estimates of effect sizes.

These sensitivity analyses greatly strengthen the inferences that in the treatment of depression tricyclics at dosages lower than the usually recommended range are more effective than placebo but possibly a little bit less effective than standard dosage tricyclics although with fewer side effects. The evidence suggests that academicians have been on weak ground in criticising clinicians' use of low dose tricyclics. Every trial protocol should include strategies for ensuring follow up of all the participants even if they stop the prescribed drug, because it is the only way to adhere to the intention to treat principle and to produce results permitting strong inferences about treatment effects. Only then can the relative benefits and harms of various dosages be definitively established.

\section{What is already known on this topic}

Tricyclics are still prescribed as often as selective serotonin reuptake inhibitors and other newer antidepressants worldwide

Experts have often claimed that clinicians prescribe tricyclics at less than adequate dosages

\section{What this study adds}

Tricyclics at dosages below the recommended range are more effective than placebo

They may or may not be as effective as standard dosage tricyclics but result in fewer dropouts due to side effects

The minimum effective dosage and ranges for antidepressants has not been established-a simple set of numbers that every practising doctor and patient would want to know and interview schedules to diagnose depression. Some studies used ad hoc outcome measures of unknown reliability and validity. Although the dropout rates were 
This systematic review was conducted within the framework of the Cochrane Collaboration Depression, Anxiety, and Neurosis Group. We thank Gordon Guyatt and David Streiner for their helpful comments on earlier drafts.

Contributors: TAF developed the idea of this systematic review and did the literature search, study selection, data extraction, and data analysis. HMcG did the literature search, study selection, and data extraction. CB did the study selection and helped in the interpretation of the results. All the investigators contributed to the writing of the paper and will act as guarantors.

Funding: St Luke's Life Science Institute, Tokyo, Japan provided 700000 yen (£3659; \$5696; €5835).

Competing interests: TAF has received fees for speaking from several pharmaceutical companies, some of which manufacture various types of antidepressants including paroxetine, fluroxamine, milnacipran.

1 Lawrenson RA, Tyrer F, Newson RB, Farmer RD. The treatment of depression in UK general practice: selective serotonin reuptake inhibitors and tricyclic antidepressants compared. J Affect Disord 2000;59:149-57.

2 Pincus HA, Tanielian TL, Marcus SC, Olfson M, Zarin DA, Thompson J, et al. Prescribing trends in psychotropic medications: primary care, psychiatry, and other medical specialties. JAMA 1998;279:526-31.

3 Sleath BL, Rubin RH, Huston SA. Antidepressant prescribing to Hispanic and non-Hispanic white patients in primary care. Ann Pharmacother 2001;35(4):419-23.
4 Roberts E, Norris P. Growth and change in the prescribing of anti-depressants in New Zealand: 1993-1997. N Z Med J 2001;114:25-7.

5 Depression Guideline Panel. Clinical practice guideline: depression in primary care 2:treatment of major depression. Rockville, MD: US Department of Heath and Human Services, Agency for Health Care Policy and Research, 1993. [AHCPR Publication 93-0551.]

6 Anderson IM, Nutt DJ, Deakin JF. Evidence-based guidelines for treating depressive disorders with antidepressants: a revision of the 1993 British Association for Psychopharmacology guidelines. British Association for Psychopharmacology. J Psychopharmacol 2000;14:3-20.

7 American Psychiatric Association. Practice guideline for the treatment of patients with major depressive disorder (Revision). Am J Psychiatry 2000;157(Apr suppl):1-45.

8 Paykel ES, Priest RG. Recognition and management of depression in general practice: consensus statement. BMJ 1992;305:1198-202.

9 Hirschfeld RM, Keller MB, Panico S, Arons BS, Barlow D, Davidoff F, et al The National Depressive and Manic-Depressive Association consensus statement on the undertreatment of depression. JAMA 1997;277:333-40.

10 Furukawa TA, Kitamura T, Takahashi K. Treatment received by depressed patients in Japan and its determinants: naturalistic observation from a multi-center collaborative follow-up study. J Affect Disord 2000:60:173-9.

11 Donoghue J, Taylor DM. Suboptimal use of antidepressants in the treatment of depression. CNS Drugs 2000;13:365-83.

12 Mulrow CD, Oxman AD. Cochrane collaboration handbook [updated 1 Mar 1997]. In: Cochrane library. Issue 4. Oxford: Update Software; 1997.

13 Furukawa TA, Guyatt GH, Grifitth LE. Can we individualize the Number Needed to Treat (NNT)? An empirical study of summary effect measures in meta-analyses. Int J Epidemiol 2002;31:72-6.

14 DerSimonian R, Charette LJ, McPeek B, Mosteller F. Reporting on methods in clinical trials. N Engl J Med 1982;306:1332-7.

(Accepted 24 June 2002) 